\title{
ON A SPLITTING THEOREM OF GASCHÜTZ
}

\author{
by JOHN S. ROSE \\ (Received 10th August 1964)
}

1. Let $G$ be any finite group, and $p$ any prime number. (All groups to be considered here are finite, and we assume this without further comment.) We denote by $K_{p}(G)$ the unique smallest normal subgroup of $G$ for which the quotient $G / K_{p}(G)$ is a p-group. $G / K_{p}(G)$ is called the p-residual of $G$. W. Gaschütz (2, Satz 7) has proved the following

Theorem. Set $K=K_{p}(G)$. If the Sylow p-subgroups of $K$ are abelian, then $G$ splits over $K$.

The method of proof adopted in (2) depends on general reduction theorems for the splitting of a group over an abelian normal subgroup, and these require quite elaborate calculations with factor systems. The techniques involved are powerful enough to yield many other interesting results, but the Theorem quoted above is perhaps of sufficient intrinsic interest to warrant the publication of another proof. This is the purpose of the present note. The chief tools employed here are the Second Theorem of Grün (7, p. 171, Theorem 6), the Theorems of Schur and Zassenhaus (7, p. 162, Theorems 25 and 27), and

Lemma 1. If the group $H$ has an abelian Sylow p-subgroup $P$, then $H^{\prime} \cap Z(H) \cap P=1$ (where $H^{\prime}$ is the derived group of $H$, and $Z(H)$ is the centre of $H$ ).

This was proved for soluble $H$ by D. R. Taunt (5), and in general by means of an easy transfer argument by B. Huppert (4).

2. Given the results mentioned above, the key to the proof of the Theorem lies in certain observations about p-complements. It is convenient to begin by introducing some notation and terminology. Suppose that $H$ is a group, and $K$ a subgroup of $H$. We write $K^{H}$ for the normal closure of $K$ in $H$, that is the unique smallest normal subgroup of $H$ which contains $K$. We write $N_{H}(K)$ for the normalizer of $K$ in $H$, that is the unique largest subgroup of $\boldsymbol{H}$ in which $K$ is contained as a normal subgroup. Thus $K$ is normal in $H$ if and only if $K^{H}=K$, and if and only if $N_{H}(K)=H$. We say that $K$ is contranormal $\dagger$ in $H$ if $K^{H}=H$; and that $K$ is self-normalizing in $H$ if $N_{H}(K)=K$. In general, $K$ may be contranormal but not self-normalizing in $H$, or self-normalizing but not contranormal in $H$; but we shall be concerned with special circumstances in which each of these properties implies the other. We may remark that the abnormal subgroups of R. W. Carter (see for instance (1)), of which normalizers of Sylow subgroups provide the most familiar example, are both contranormal and self-normalizing.

$\dagger$ This term has been introduced by Professor P. Hall. 
A subgroup $Q$ is called a p-complement of a group $H$ if $Q$ has order prime to $p$, and index in $H$ a power of $p$. In general a group need not possess a $p$-complement. However, we note the obvious fact that if a group $H$ has a p-complement $Q$, then $Q^{H}=K_{p}(H)$. In particular, $Q$ is contranormal in $H$ if and only if $K_{p}(H)=H$, that is if and only if $H$ has trivial $p$-residual. A group with trivial $p$-residual is said to be $p$-perfect.

By Schur's Theorem, a sufficient condition for the existence of a $p$-complement in a group $H$ is that $H$ has a normal Sylow p-subgroup $P$, for then $H$ splits over $P$; and in that case Zassenhaus's Theorem shows that the $p$-complements form a single conjugacy class of subgroups in $H$. We deduce

Lemma 2. If $H$ is a normal subgroup of the group $G$, and $H$ has a normal Sylow p-subgroup, then $G=H N_{G}(Q)$, where $Q$ is any p-complement of $H$.

Proof. This goes by the usual Frattini argument. For any $g \in G$, we have $g^{-1} Q g \leqq H$; and then, since $g^{-1} Q g$ is a $p$-complement of $H$, we know that there exists $h \in H$ such that $g^{-1} Q g=h^{-1} Q h$. Then $g h^{-1} \in N_{G}(Q)$, and the result follows.

Now suppose that the group $H$ has a normal Sylow $p$-subgroup, and let $Q$ be a $p$-complement of $H$. It follows from the fact that the $p$-complements form a single conjugacy class in $H$ that $N_{H}(Q)$ is abnormal in $H$-by an argument exactly similar to the one used in proving that the normalizer of a Sylow subgroup is abnormal in a group. Hence if $Q$ is self-normalizing in $H$, then $Q$ is abnormal in $H$, and so in particular $Q$ is contranormal in $H$. The converse is false: a group $H$ may have a normal Sylow $p$-subgroup, and a $p$-complement $Q$ which is contranormal but not self-normalizing in $H$. For instance, in a split extension of a quaternion group by a cyclic group of order 3 , defined by means of an automorphism permuting cyclically the three subgroups of order 4 in the quaternion group, a 2-complement is contranormal, but is of index 2 in its normalizer. However, we shall see that the imposition of an extra condition makes this converse true.

Lemma 3. Suppose that the group $H$ has a normal abelian Sylow p-subgroup $P$, and let $Q$ be a p-complement of $H$. If $Q$ is contranormal in $H$, then $Q$ is self-normalizing in $H$.

Proof. Let $P_{0}=P \cap N_{H}(Q)$. Since $P$ is normal in $H, P_{0}$ is normal in $N_{H}(Q)$. Since also $Q$ is normal in $N_{H}(Q)$ and $P_{0} \cap Q=1, N_{H}(Q)=P_{0} \times Q$ (direct product), and $P_{0}$ centralizes $Q$. Moreover, $P_{0}$ centralizes $P$, since $P$ is abelian. Therefore $P_{0}$ centralizes $P Q=H$, that is $P_{0} \leqq Z(H)$, the centre of $H$.

Now by hypothesis $Q^{H}=H$, or equivalently, $H$ is $p$-perfect. It follows from this that $P \leqq H^{\prime}$, the derived group of $H$. Hence $P_{0} \leqq H^{\prime} \cap Z(H) \cap P=1$, by Lemma 1 . Therefore $N_{H}(Q)=Q$.

\section{Proof of the Theorem}

We proceed by induction on the group order. Let $P$ be a Sylow $p$-subgroup of $K$. $P$ is abelian, so that $K$ is $p$-normal in the sense of Grün. By definition 
of $K, K$ is $p$-perfect, and therefore the Second Theorem of Grün shows that $N_{K}(P)$ is $p$-perfect. Let $N=N_{G}(P)$; then $N \cap K=N_{K}(P)$. $N \cap K$ is normal in $N$, and since $N / N \cap K$ is isomorphic to $N K / K, N / N \cap K$ is a $p$-group. Since we have shown that $N \cap K$ is $p$-perfect, we must have $N \cap K=K_{p}(N)$. As a subgroup of $K, N \cap K$ has abelian Sylow $p$-subgroups. Hence if $N$ is a proper subgroup of $G$, the induction hypothesis implies that $N$ splits over $N \cap K$, say $N=$ $(N \cap K) P^{*}$ with $(N \cap K) \cap P^{*}=1$. Then, since $P^{*} \leqq N, K \cap P^{*}=1$. Therefore, since the Frattini argument shows that $G=K N$, we have $G=K P^{*}$ with $K \cap P^{*}=1$. Thus $G$ splits over $K$.

So we may suppose now that $N=G$, that is that $P$ is normal in $G$. Then $P$ is normal in $K$, so that by Schur's Theorem, $K$ possesses a $p$-complement $Q$. $K$ is $p$-perfect, and so $Q^{K}=K$. Since $P$ is abelian, it follows from Lemma 3 that $N_{K}(Q)=Q$.

Now by Lemma 2, $G=K N_{G}(Q)$. Let $P^{*}$ be a Sylow $p$-subgroup of $N_{G}(Q)$, so that $N_{G}(Q)=Q P^{*}$. Then, since $Q \leqq K, G=K P^{*}$. Also

$$
Q=N_{K}(Q)=K \cap N_{G}(Q)=K \cap\left(Q P^{*}\right)=Q\left(K \cap P^{*}\right) \text {, }
$$

so that as $K \cap P^{*}$ is a $p$-group contained in $Q$, which has order prime to $p$, we must have $K \cap P^{*}=1$. Thus $G$ splits over $K$. This completes the induction argument.

4. P. Hall $(3, \S 5)$ has described examples of groups which show that the condition that the Sylow $p$-subgroups of $K$ be abelian is indispensable for the truth of the Theorem. For instance, let $X$ be a non-abelian group of order 27 and exponent 3. The centre $Z$ of $X$ has order 3. The automorphism group of $X$ has a quaternion subgroup $Q$ which leaves $Z$ invariant. If we form a split extension $H$ of $X$ by means of $Q$, and then extend $H$ by an element $a$ commuting with every element of $H$ and such that $a^{3}$ generates $Z$, we obtain a group $G$ of order 648. $K_{3}(G)=H$, but $H$ contains all elements of $G$ of order 3, so that $G$ cannot split over $H$.

5. In this final section, we add some remarks on a particular application of the Theorem. A group $G$ is said to be $p$-nilpotent if $K_{p}(G)$ has order prime to $p$ (in which case $K_{p}(G)$ is the unique $p$-complement in $G$ ). Thus the extremes of behaviour for a group, in regard to its $p$-residual, are to be $p$-nilpotent or $p$-perfect. The Theorem has the following

Corollary. If $G$ is a group with cyclic Sylow p-subgroups, then either $G$ is p-nilpotent or $G$ is p-perfect.

Proof. Let $K=K_{p}(G)$. The hypothesis for $G$ is inherited by subgroups, so that the Sylow $p$-subgroups of $K$ are cyclic. Hence by the Theorem, $G$ splits over $K$, and so there is a $p$-subgroup $P$ of $G$ with $G=K P$ and $K \cap P=1$. Let $\bar{P}$ be a Sylow $p$-subgroup of $G$ containing $P . K \cap \bar{P}$ is normal in $\bar{P}$, and since $G=K P, \bar{P}=(K \cap \bar{P}) P ;$ and of course $(K \cap \bar{P}) \cap P=1$. Since $\bar{P}$ is cyclic, it follows that either $P=\bar{P}$ or $P=1$. This gives the result.

Further properties of groups with cyclic Sylow $p$-subgroups have been obtained by $\mathrm{H}$. Wielandt $(6, \S 4)$. 
We may note that if $p=2$ in this Corollary, then $G$ is actually 2-nilpotent. This may be proved by an elementary argument, as follows.

Proposition. If $G$ is a group with cyclic Sylow 2-subgroups, then $G$ is 2-nilpotent.

Proof. Let $\rho$ be the right regular representation of $G$. Suppose that $G$ has order $2^{m} n$, where $n$ is odd and we may assume that $m>0$. Let $x$ be a generator of a Sylow 2-subgroup of $G$. Then $\rho(x)$ may be expressed as a product of $n$ disjoint cycles of equal length $2^{m}$, and hence $\rho(x)$ is an odd permutation. Therefore the even permutations form a subgroup of index 2 in $\rho(G)$. By the isomorphism between $G$ and $\rho(G), G$ has a subgroup $G_{1}$ of index 2. If $G_{1}$ has even order, $G_{1}$ is again a group with cyclic Sylow 2-subgroups, so that we may repeat the argument above to show that $G_{1}$ has a subgroup $G_{2}$ of index 2 . Repetition of the argument yields a chain of subgroups

$$
G=G_{0}>G_{1}>\ldots>G_{m},
$$

with $G_{i}$ of index 2 in $G_{i-1}$ for each $i=1, \ldots, m . G_{m}$ has odd order. By induction on $m$, we show that $G_{m}$ is normal in $G$, so that $G_{m}=K_{2}(G)$ and $G$ is 2-nilpotent.

Remarks. $1^{0}$. Since groups of odd order are soluble (Feit-Thompson Theorem), we may deduce from this result that a group with cyclic Sylow 2 -subgroups is necessarily soluble.

$2^{\circ}$. If, in place of the elementary argument used above, we apply the Theorem of Burnside which asserts that if a group $G$ has a Sylow $p$-subgroup in the centre of its normalizer, then $G$ is p-nilpotent, we can obtain the following stronger result. If $G$ is a group with cyclic Sylow $p$-subgroups, where $p$ is the least prime factor of the order of $G$, then $G$ is $p$-nilpotent. This is known.

\section{REFERENCES}

(1) R. W. CARTER, Nilpotent self-normalizing subgroups of soluble groups, Math. Zeitschrift, 75 (1961), 136-139.

(2) W. Gaschütz, Zur Erweiterungstheorie der endlichen Gruppen, J. reine angew. Math., 190 (1952), 93-107.

(3) P. Hall, The splitting properties of relatively free groups, Proc. London Math. Soc., (3) 4 (1954), 343-356.

(4) B. HuPPERT, Subnormale Untergruppen und p-Sylowgruppen, Acta Sci. Math. Szeged, 22 (1961), 46-61.

(5) D. R. Taunt, On A-groups, Proc. Cambridge Phil. Soc., 45 (1949), 24-42.

(6) H. Wielandt, Sylowgruppen und Kompositions-Struktur, Abh. Math. Sem. Hamburg, 22 (1958), 215-228.

(7) H. J. Zassenhaus, The Theory of Groups, 2nd ed. (New York, 1958).

\section{Peterhouse}

CAMBridge 